by small amounts of blue light, can be initiated by treating the fungus with a mixture of acetylcholine and the acetylcholinesterase inhibitor, eserine.

That acetylcholine, known chiefly for its role in the transmission of nerve impulses in animals, may be active in plant photomorphogenesis is a recent and, on the face of things, bizarre idea. But acetylcholine is known from animal studies to mediate certain processes involving changes in membrane permeability, and it is just these sorts of changes which many plant physiologists believe to be the basis of some aspects of photomorphogenesis.

M. J. Jaffe showed last year that mung bean seedlings not only contain appreciable quantities of a substance which the results of a series of tests suggested strongly to be acetylcholine, but also that the levels of acetylcholine in the plant changed characteristically after exposure to red light. Furthermore, acetylcholine, when given to dark grown seedlings, mimicked the effect of red light in certain photomorphogenic responses. One of these responses was the odd electrical phenomenon discovered by Tanada, where mung bean root tips cling to a negatively charged wet glass surface in red light and release in far-red light (see Nature New Biology, 230, 257 ; 1971). The implication is that acetylcholine is involved in electrical changes in the plant which presumably follow the photoconversion of phytochrome.

The results that Gressel et al. have obtained for Trichoderma are not so satisfying as Jaffe's for mung bean, perhaps, but they do imply that there may be a fundamental similarity underlying some red and blue light induced photoresponses. Trichoderma sporulates in culture after exposure to blue light but not in darkness. Acetylcholine alone cannot replace blue light in this system, but Gressel et al. were able to induce sporulation in the dark using acetylcholine in combination with eserine. The relative amounts needed were specific; sporulation equivalent to 95 per cent of the control value was obtained when $10 \times 10^{-5} \mathrm{M}$ acetylcholine was used with $5 \times 10^{-6} \mathrm{M}$ eserine. Curare, an acetylcholine antagonist, suppressed sporulation in the light control material.

Gressel and his colleagues, however, have not indicated very clearly how they think their data applies to the natural regulation of photomorphogenesis. Is, for example, acetylcholine found naturally in Trichoderma, and if so what changes take place after exposure to blue or red light? Does addition of acetylcholine and eserine to the fungus really step round the mechanism controlling sporulation or merely provide the operating factors for altering membrane permeability? The authors tentatively suggest that should acetyl- choline prove common to the mechanism of both blue and red light photomorphogenesis, their modes of action will have to be thought out anew. Very true; but these latest results say less about this prospect than raise questions about the basic nature of the changes which lead to sporulation in blue light sensitive fungi.

\section{RHIPIDISTIA \\ Links with Tetrapods}

from our Vertebrate Palaeontology Correspondent

IN the study of the course of vertebrate evolution, one of the most important groups of animals are the extinct rhipidistian fishes. Rhipidistia first appear in the fossil record in the early Devonian and persist into the Permian and it is generally agreed that this group gave rise to all the tetrapods-amphibia, reptiles, birds and mammals. Important information on the anatomy of these forms is contained in two recent articles written jointly by S. M. Andrews and T. W. Westoll (Trans. Roy. Soc. Edinb., 68, Nos. 9 and 12 ; 1971).

As would be expected vertebrate palaeontologists have devoted considerable time to the Rhipidistia, but detailed study has been largely confined to de- scription of the skull and speculations about their relationships have been based almost exclusively on cranial anatomy. This is very strikingly the case with Eusthenopteron. This fish is known chiefly from the early Upper Devonian of Canada and has been a focus of attention since the early years of this century. The skull is probably better known than that of any other fossil vertebrate, and has been studied particularly by Jarwik who has used the technique of serial grinding originally perfected in Sweden by Stensiö. Although there have been many partial accounts of the rest of the skeleton, a comprehensive, detailed and accurate description of the rest of the skeleton has been lacking, so that the work of Andrews and Westoll on the postcranial skeleton of Eusthenopteron is welcome.

In their first article, a description of the paired pectoral fins is accompanied by a detailed analysis of the probable muscle origins and insertions of the fins and shoulder girdle. The resemblance of the proximal part of the fin to the primitive tetrapod limb was already known in general terms; detailed analysis now shows that the range of movements of the early tetrapod humerus is almost identical to that of the first axial element of the fin of Eusthenopteron. This part of the rhipidistian fin seems in

\title{
Structural Gene of Diphtheria Toxin
}

IN next Wednesday's Nature New Biology, Uchida, Gill and Pappenheimer report an elegant series of experiments which prove that the structural gene for diphtheria toxin is carried by the lysogenic bacteriophage $\beta$ and some of its close relatives. It has, of course, been known for several years that strains of the diphtheria bacillus Corynebacterium diphtheriae which do not make the toxin are rendered toxinogenic when they are lysogenized by bacteriophage $\beta$ and that the yield of toxin depends on the strain of bacterium and its physiological state. Moreover, the tox character of phage $\beta$ maps at a single locus.

In an attempt to decide between the two alternative explanations of these findings, namely that the tox gene specifies the toxin protein or that it regulates the expression of a structural gene for the toxin in the bacterial chromosome, Uchida et al. devised an interesting selection procedure which enabled them to isolate a mutant of phage $\beta$. Cells of non-toxinogenic strains of $C$. diphtheriae lysogenized by this mutant phage make a protein which cross reacts with anti-toxin antibody but is not toxic and lacks the $\mathbf{C}$ terminal sequence of the normal toxin molecule. Clearly, therefore, the tox gene specifies the toxin protein itself and the mutation
Uchida et al. have isolated is either a deletion or a nonsense mutation which prematurely terminates the synthesis of toxin protein.

These findings lead Uchida et al. to speculate about the evolution of the tox gene. Diphtheria toxin, the substrate of which is restricted to eukaryotic cells, has no role in the replication of phage $\beta$ and its relatives; it can, for example, be totally deleted without altering the rate of phage replication or the yield of phage. Neither does the toxin have any apparent role in the life cycle of the diphtheria bacillus growing in culture.

Why then has the gene been retained? Uchida et al. suggest that the ability to make toxin must have some survival value for the bacillus, and therefore its lysogenic phages, in its natural habitat - the throats of animals and man-and they point out that widespread immunization with diptheria toxoid has resulted in the eradication of toxinogenic strains of $C$. diphtheriae from many human populations. As for the origin of the toxin gene, Uchida and his colleagues speculate that because the only known substrate of the toxin is found exclusively in eukaryotic cells, the toxin gene may have once been a eukaryotic gene which the bacilli or their phage picked up and modified to their own advantage. 